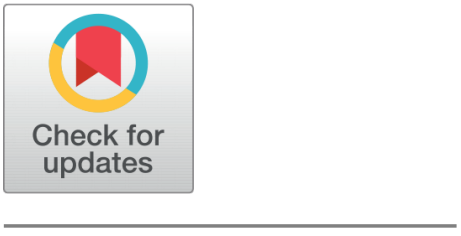

OPEN ACCESS

Received: 22.12 .2020

Accepted: 02.02.2021

Published: 16.02.2021

Citation: Reddy GU, Rao TSN, Rao NS, Rao VV (2021) Bipolar single valued neutrosophic detour distance. Indian Journal of Science and Technology 14(5): 427-431. http s://doi.org/10.17485/IJST/v14i5.2102

* Corresponding author.

yuviganga@gmail.com

Funding: None

Competing Interests: None

Copyright: (c) 2021 Reddy et al. This is an open access article distributed under the terms of the Creative Commons Attribution License, which permits unrestricted use, distribution, and reproduction in any medium, provided the original author and source are credited.

Published By Indian Society for Education and Environment (iSee)

ISSN

Print: 0974-6846

Electronic: 0974-5645

\section{Bipolar single valued neutrosophic detour distance}

\author{
G Upender Reddy ${ }^{*}$, T Siva Nageswara Rao ${ }^{2}$, N Srinivasa Rao ${ }^{3}$, \\ V Venkateswara Rao4
}

1 Assistant Professor, Department of Mathematics, Nizam College (A), Osmania University, Hyderabad, 500 001, TS, India

2 Associate Professor, Division of Mathematics, Department of S\&H, V.F. S.T. R (Deemed to be University), Vadlamudi, 522 207, Guntur (Dt.), A.P, India

3 Assistant Professor, Division of Mathematics, Department of S\&H, V.F. S.T. R (Deemed to be University), Vadlamudi, 522 207, Guntur (Dt.), A.P, India

4 Research Scholar, Division of Mathematics, Department of S\&H, V.F. S.T. R (Deemed to be University), Vadlamudi, 522 207, Guntur (Dt.), A.P, India

\section{Abstract}

Objectives: In the present article, we deduced a characterization of Bipolar Single Valued Neutrosophic (BSVN) radius and eccentricity of the vertex based on Bipolar Single Valued Neutrosophic set(BSVNS) detour. Method: We obtained some definitions BSVN on a vertex like BSVN detour eccentric vertex, BSVN detour radius, BSVN detour diameter, BSVN detour centered and BSVN detour periphery. Findings: We derived some important results based on these BSVN detour radius, diameter, center and periphery. Novelty: The detour distance of the BSVNS model is proposed and generalized by this. An important and suitable condition for the graphs of the Single Valued Neutrosophic Set(SVNS) model to BSVNS detour distances has been identified.

Keywords: Detour distance; BSVN detour eccentric; BSVN detour distance; BSVN detour peripheral node; BSVN detour path

\section{Introduction}

The Neutrosophic sets ${ }^{(1)}$ is a great exact implement for the situation uncertainty in the real world. These uncertainty ideas come from the theories of fuzzy sets ${ }^{(2)}$, intuitionistic fuzzy sets ${ }^{(3)}$ and interval-valued intuitionistic fuzzy sets ${ }^{(4)}$. The representation of the neutrosophic sets is truth, indeterminacy and falsity value. These T, I, F values belong to standard or nonstandard unit interval denoted by $]-0,1+[$.

The idea of a subclass of the NS and SVNS by intuitionistic fuzzy sets ${ }^{(5)}$, in this the functions Truth, Indeterminacy, Falsity are not dependent and these values are present within $[0,1]$. Neutrosophic theory is widely expanded in all fields especially in Graph theory and Topology.

In a graph theory, the new graph model was invited by using BSVN set is known as BSVN Graph (BSVNG) ${ }^{(6,7)}$. The idea of BSVN graphs from the fuzzy, bipolar fuzzy and single-valued neutrosophic graphs. The uncertainty on the graph of vertices and edges or both representations to be a fuzzy graph. 
In this manuscript, we discuss about BSVN graphs and neutrosophic detour distance between two vertices of the graph based on BSVN eccentricity, radius, diameter, and the periphery with respect to detour distance.

\section{Preliminaries}

\section{Explanation 2.1: BSVN sets}

A BSVN set is explained as the membership functions represented as an object in $W$ is denoted by $\left\{<w, T^{P}, I^{P}, F^{P}, T^{N}, I^{N}, F^{N}>: w \varepsilon W\right\}$ the functions $T^{P}, I^{P}, F^{P}$ are mapping from $W$ to $[0,1]$ and $T^{N}, I^{N}, F^{N}$ are mapping from $W$ to $[-1,0]$.

Example. Let $W=\left\{w_{1}, w_{2}, w_{3}\right\}$ and $A=\left\{\left\langle w_{1}, 0.4,0.2,0.6,-0.03,-0.2,-0.01\right\rangle,<w_{2}, 0.6,0.4,0.2,-0.5,-0.3,-0.03\right\rangle$,

$\left.<w_{3}, 0.7,0.04,0.3,-0.8,-0.4,-0.05>\right\}$ is a BSVN set in $W$.

\section{Explanation 2.2: SVN relation on $W$ :-}

Let $W$ be a non-empty set. Then we call mapping $Z=\left(W, T^{p}, I^{p}, F^{P}, T^{N}, I^{N}, F^{N}\right), F^{N}(w): W X W \rightarrow[-1,0] X[0,1]$ is a BSVN relation on $W$ such that

$T_{Z}^{P}\left(w_{1}, w_{2}\right) \varepsilon[0,1], I_{Z}^{P}\left(w_{1}, w_{2}\right) \varepsilon[0,1], F_{Z}^{P}\left(w_{1}, w_{2}\right) \varepsilon[0,1]$ and $T_{Z}^{N}\left(w_{1}, w_{2}\right) \varepsilon[-1,0], I_{Z}^{N}\left(w_{1}, w_{2}\right) \varepsilon[-1,0], F_{Z}^{N}\left(w_{1}, w_{2}\right) \varepsilon[-1,0]$

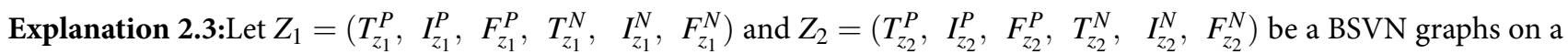
set $W$. If $Z_{2}$ is a BSVN relation on $Z_{1}$, then

$T_{Z_{2}}^{P}\left(w_{1}, w_{2}\right) \leq \min \left(T_{Z_{1}}^{P}\left(w_{1}\right), T_{Z_{1}}^{P}\left(w_{2}\right)\right), I_{Z_{2}}^{P}\left(w_{1}, w_{2}\right) \geq \max \left(I_{Z_{1}}^{P}\left(w_{1}\right), I_{Z_{1}}^{P}\left(w_{2}\right)\right), F_{Z_{2}}^{P}\left(w_{1}, w_{2}\right) \geq \max \left(F_{Z_{1}}^{P}\left(w_{1}\right), F_{Z_{1}}^{P}\left(w_{2}\right)\right)$ and $T_{Z_{2}}^{N}\left(w_{1}, w_{2}\right) \geq \max \left(T_{Z_{1}}^{N}\left(w_{1}\right), T_{Z_{1}}^{N}\left(w_{2}\right)\right), I_{Z_{2}}^{P}\left(w_{1}, w_{2}\right) \leq \min \left(I_{Z_{1}}^{N}\left(w_{1}\right), I_{Z_{1}}^{N}\left(w_{2}\right)\right), F_{Z_{2}}^{N}\left(w_{1}, w_{2}\right) \leq \min \left(F_{Z_{1}}^{N}\left(w_{1}\right), F_{Z_{1}}^{N}\left(w_{2}\right)\right)$ for all $w_{1}, w_{2} \in W$

Explanation 2.4: The symmetric property defined on BSVN relation $Z$ on $W$ is explained by

$T_{Z}^{P}\left(w_{1}, w_{2}\right)=T_{Z}^{P}\left(w_{2}, w_{1}\right), I_{Z}^{P}\left(w_{1}, w_{2}\right)=I_{Z}^{P}\left(w_{2}, w_{1}\right), F_{Z}^{P}\left(w_{1}, w_{2}\right)=F_{Z}^{P}\left(w_{2}, w_{1}\right)$ and $T_{Z}^{N}\left(w_{1}, w_{2}\right)=T_{Z}^{N}\left(w_{2}, w_{1}\right)$, $I_{Z}^{N}\left(w_{1}, w_{2}\right)=I_{Z}^{N}\left(w_{2}, w_{1}\right), F_{Z}^{N}\left(w_{1}, w_{2}\right)=F_{Z}^{N}\left(w_{2}, w_{1}\right)$ for all $w_{1}, w_{2} \in W$

\section{Explanation 2.5 : BSVN graph}

The new graph in SVN is denoted by for all $w_{1}, w_{2} \in W, G^{*}=(V, E)$ is a pair $G=\left(Z_{1}, Z_{2}\right)$, where $Z_{1}=$ $\left(T_{z_{1}}^{P}, I_{z_{1}}^{P}, F_{z_{1}}^{P}, T_{z_{1}}^{N}, I_{z_{1}}^{N}, F_{z_{1}}^{N}\right)$ is a BSVNS in $V$ and $Z_{2}=\left(T_{z_{2}}^{P}, I_{z_{2}}^{P}, F_{z_{2}}^{P}, T_{z_{2}}^{N}, I_{z_{2}}^{N}, F_{z_{2}}^{N}\right)$ is BSVNS in $V^{2}$ defined as

$T_{Z_{2}}^{P}\left(w_{1}, w_{2}\right) \leq \min \left(T_{Z_{1}}^{P}\left(w_{1}\right), T_{Z_{1}}^{P}\left(w_{2}\right)\right), I_{Z_{2}}^{P}\left(w_{1}, w_{2}\right) \geq \max \left(I_{Z_{1}}^{P}\left(w_{1}\right), I_{Z_{1}}^{P}\left(w_{2}\right)\right), F_{Z_{2}}^{P}\left(w_{1}, w_{2}\right) \geq \max \left(F_{Z_{1}}^{P}\left(w_{1}\right), F_{Z_{1}}^{P}\left(w_{2}\right)\right)$ and $T_{Z_{2}}^{N}\left(w_{1}, w_{2}\right) \geq \max \left(T_{Z_{1}}^{N}\left(w_{1}\right), T_{Z_{1}}^{N}\left(w_{2}\right)\right), I_{Z_{2}}^{P}\left(w_{1}, w_{2}\right) \leq \min \left(I_{Z_{1}}^{N}\left(w_{1}\right), I_{Z_{1}}^{N}\left(w_{2}\right)\right), F_{Z_{2}}^{N}\left(w_{1}, w_{2}\right) \leq \min \left(F_{Z_{1}}^{N}\left(w_{1}\right), F_{Z_{1}}^{N}\left(w_{2}\right)\right)$ for all $w_{1}, w_{2} \in W$

The BSVNSG of an edge denoted by $w_{1} w_{2} \in V^{2}$

Explanation 2.6 Let $G=\left(Z_{1}, Z_{2}\right)$ be a BSVNSG and $a, b \in V$. A path $P: a=w_{0}, w_{1}, w_{2}, \ldots, w_{k-1}, w_{k}=b$ in $G$ is a sequence of distinct vertices such that $T_{Z_{2}}^{P}\left(w_{i-1}, w_{i}\right)>0, I_{Z_{2}}^{P}\left(w_{i-1}, w_{i}\right)>0, F_{Z_{2}}^{P}\left(w_{i-1}, w_{i}\right)>0, T_{Z_{2}}^{N}\left(w_{i-1}, w_{i}\right)>0$, $I_{Z_{2}}^{N}\left(w_{i-1}, w_{i}\right)>0, F_{Z_{2}}^{N}\left(w_{i-1}, w_{i}\right)>0$ where $i=1,2,3, \ldots, k$ and length of the path is $k \in N($ a positive integer $)$, where $a$ is called initial vertex and $b$ is called terminal vertex in the path.

Explanation 2.7. A BSVN graph $G=\left(Z_{1}, Z_{2}\right)$ of $G^{*}=(V, E)$ is called a strong BSVN graph if $T_{Z_{2}}^{P}\left(w_{1}, w_{2}\right)=$ $\min \left(T_{Z_{1}}^{P}\left(w_{1}\right), T_{Z_{1}}^{P}\left(w_{2}\right)\right), I_{Z_{2}}^{P}\left(w_{1}, w_{2}\right)=\max \left(I_{Z_{1}}^{P}\left(w_{1}\right), I_{Z_{1}}^{P}\left(w_{2}\right)\right)$,

$F_{Z_{2}}^{P}\left(w_{1}, w_{2}\right)=\max \left(F_{Z_{1}}^{P_{1}}\left(w_{1}\right), \quad F_{Z_{1}}^{P}\left(w_{2}\right)\right) \quad$ and $T_{Z_{2}}^{N}\left(w_{1}, w_{2}\right)=\max \left(T_{Z_{1}}^{N}\left(w_{1}\right), \quad T_{Z_{1}}^{N}\left(w_{2}\right)\right), I_{Z_{2}}^{P}\left(w_{1}, w_{2}\right)=$ $\min \left(I_{Z_{1}}^{N}\left(w_{1}\right), I_{Z_{1}}^{N}\left(w_{2}\right)\right), F_{Z_{2}}^{N}\left(w_{1}, w_{2}\right)=\min \left(F_{Z_{1}}^{N}\left(w_{1}\right), F_{Z_{1}}^{N}\left(w_{2}\right)\right)$ for all $w_{1}, w_{2} \in W$ 
If $P: a=w_{0}, w_{1}, w_{2}, \ldots, w_{k-1}, w_{k}=b$ be a path of length $k$ between $a$ and $b$ then $\left(T_{Z_{2}}^{P}(a, b), I_{Z_{2}}^{P}(a, b), F_{Z_{2}}^{P}(a, b)\right)^{k}$ and $\left(T_{Z_{2}}^{N}(a, b), I_{Z_{2}}^{N}(a, b), F_{Z_{2}}^{N}(a, b)\right)^{k}$ is defined as

$$
\begin{aligned}
& \left(T_{Z_{2}}^{P}(a, b), I_{Z_{2}}^{P}(a, b), F_{Z_{2}}^{P}(a, b)\right)^{k}= \begin{cases}\sup \left\{T _ { Z _ { 2 } } ^ { P } ( a , w _ { 1 } ) \wedge T _ { Z _ { 2 } } ^ { P } ( w _ { 1 } , w _ { 2 } ) \wedge \ldots \wedge T _ { Z _ { 2 } } ^ { P } \left(w_{k-1},\right.\right. & b\} \\
\inf \left\{I _ { Z _ { 2 } } ^ { P } ( a , w _ { 1 } ) \vee I _ { Z _ { 2 } } ^ { P } ( w _ { 1 } , w _ { 2 } ) \vee \ldots \vee I _ { Z _ { 2 } } ^ { P } \left(w_{k-1},\right.\right. & b\} \\
\inf \left\{F _ { Z _ { 2 } } ^ { P } ( a , w _ { 1 } ) \vee F _ { Z _ { 2 } } ^ { P } ( w _ { 1 } , w _ { 2 } ) \vee \ldots \vee F _ { Z _ { 2 } } ^ { P } \left(w_{k-1},\right.\right. & b\}\end{cases}
\end{aligned}
$$

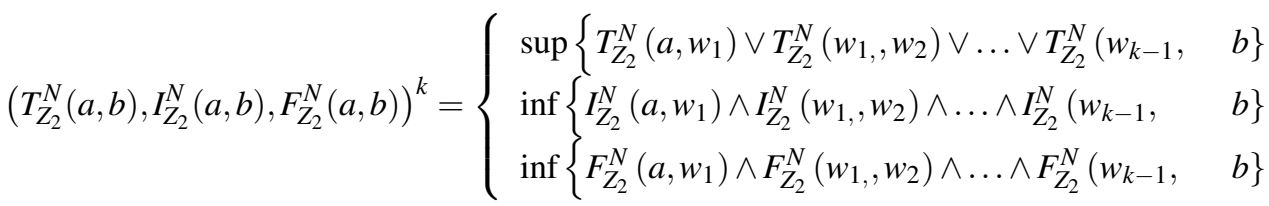

$\left(T_{Z_{2}}^{P}(a, b), I_{Z_{2}}^{P}(a, b), F_{Z_{2}}^{P}(a, b)\right)^{\infty}$ and $\left(T_{Z_{2}}^{N}(a, b), I_{Z_{2}}^{N}(a, b), F_{Z_{2}}^{N}(a, b)\right)^{\infty}$ is said to be the strength of connectedness between two vertices $x$ and $y$ in $\mathrm{G}$, where

$$
\begin{gathered}
\left(T_{Z_{2}}^{P}(a, b), I_{Z_{2}}^{P}(a, b), F_{Z_{2}}^{P}(a, b)\right)^{\infty}=\left(\sup \left\{T_{Z_{2}}^{P}(a, b)\right\}, \inf \left\{T_{Z_{2}}^{P}(a, b)\right\}, \inf \left\{F_{z_{2}}^{P}(a, b)\right\}\right) \\
\left(T_{Z_{2}}^{N}(a, b), I_{Z_{2}}^{N}(a, b), \quad F_{Z_{2}}^{N}(a, b)\right)^{\infty}=\left(\operatorname{in} f\left\{T_{Z_{2}}^{N}(a, b)\right\}, \sup \left\{T_{Z_{2}}^{N}(a, b)\right\}, \sup \left\{F_{z_{2}}^{N}(a, b)\right\}\right)
\end{gathered}
$$

If $\left(T_{Z_{2}}^{P}(a, b) \geq\left(T_{Z_{2}}^{P}(a, b)\right)^{\infty}, \quad I_{Z_{2}}^{P}(a, b) \leq\left(I_{Z_{2}}^{P}(a, b)\right)^{\infty}, F_{Z_{2}}^{P}(a, b) \leq\left(F_{Z_{2}}^{P}(a, b)\right)^{\infty}\right)$ and

$\left(T_{Z_{2}}^{N}(a, b) \geq\left(T_{Z_{2}}^{N}(a, b)\right)^{\infty}, \quad I_{Z_{2}}^{N}(a, b) \leq\left(I_{Z_{2}}^{N}(a, b)\right)^{\infty}, F_{Z_{2}}^{N}(a, b) \leq\left(F_{Z_{2}}^{N}(a, b)\right)^{\infty}\right)$ then the arc ab in $\mathrm{G}$ is called a strong arc. A path $a-b$ is a strong path if all arcs on the path are strong.

\section{BSVN detour distance}

\section{Explanation 3.1}

BSVN detour distance is defined as the length of $a-b$. The strong path between $a$ and $b$ is if there is no other path longer than $\mathrm{P}$ between $a$ and $b$ and we denote this by $\operatorname{BSND}(a, b)$. Any a-b strong path whose length is BSND $(a, b)$ is called an $a-b$ BSVN detour path. The eccentricity $\left(\operatorname{Ecc}_{B N D}(G)\right)$ of a node is the distance from a node to the furthest node in the bipolar single-valued neutrosophic graph $\mathrm{G}$. The $\operatorname{radius}\left(\operatorname{rad}_{B N G}(G)\right)$ of a bipolar single-valued neutrosophic graph $\mathrm{G}$ is the minimum among all eccentricity of nodes. The periphery of the graph is the eccentricity equal to the diameter of the path. The diameter $\left(\operatorname{diam}_{B N D}(G)\right)$ of a bipolar single valued neutrosophic graph $\mathrm{G}$ is the maximum among all eccentricity of nodes.

\section{BSVN detour periphery $\left(\operatorname{Per}_{B N D}(\mathbf{G})\right)$ and BSVN detour eccentric graph $\left(\operatorname{Ecc}_{B N D}(\mathbf{G})\right)$}

Theorem 4.1 A BSVN graph G is a BSVN detour self-centered if and only if every node of G is a BSVN detour eccentric.

Proof. Suppose G is a BSVN detour self-centered BSVN graph and let b be a node in G.

Let $a \in b_{B N D}^{*}$. So $\operatorname{Ecc}_{B N D}(b)=B N D_{G}(a, b)$. Since $\mathrm{G}$ is a BSVN detour self-centered BSVN graph, $E c c_{B N D}(a)=$ $\operatorname{Ecc}_{B N D}(b)=B N D_{G}(a, b)$ and this implies that $b \in a_{B N D}^{*}$. Hence $b$ is a BSVN detour eccentric node of G.

Conversely, let every node of G is a BSVN detour eccentric node. If possible, let G be not BSVN detour self centrad BSVN graph. Then $\operatorname{rad}_{B N D}(G) \neq \operatorname{diam}_{B N D}(G)$ and there exist node $r \in G$ such that $\operatorname{Ecc}_{B N D}(r)=\operatorname{diam}_{B N D}(G)$. Also, let $P \in r_{B N D}^{*}$. Let $U$ be $r-p$ BSVN detour in G. So there must have a node q on $\mathrm{U}$ for which the node q is not a BSVN detour eccentric node of $U$. Also, $q$ cannot be a BSVN detour eccentric node of every other node. Again if $q$ be a BSVN detour eccentric node of a node $a$ (say), this means $q \in a_{B N D}^{*}$. Then there exists an extension of $a-q$ BSVN detour up to $r$ or up to $p$. But this contradicts the facts that $q \in a_{B N D}^{*}$. Hence, $\operatorname{rad}_{B N D}(G)=\operatorname{diam}_{B N D}(G)$ and G is a BSVN detour self-centered BSVN graph.

Theorem 4.2. If $G$ is a BSVN detour self-centered BSVN graph, then $\operatorname{rad}_{B N D}(G)=\operatorname{diam}_{B N D}(G)=n-1$, where $n$ is the number of nodes of $G$. 
Proof. Let G be a BSVN detour self-centered BSVN graph.

If possible, let $\operatorname{diam}_{B N D}(G)=l<n-1$. Let $U_{1}$ and $U_{2}$ be two distinct BSVN detour peripheral path. Let $p \in U_{1}, q \in U_{2}$. So there exist a strong path between $p$ and $q$, because of the connectedness of G. Then there exist nodes on $U_{1}$ and $U_{2}$, whose eccentricity $>l$, but this is impossible, because $\operatorname{diam}_{B N D}(G)=l$. Hence $U_{1}$ and $U_{2}$ are not distinct. Since $U_{1}$ and $U_{2}$ are arbitrary, so there exist node $r$ in G such that $r$ is common in all BSVN detour peripheral paths. So $E_{c c} c_{B N D}(r)<l$, which is impossible, because G is a BSVN detour self-centered. Hence, $\operatorname{diam}_{B N D}(G)=n-1=\operatorname{rad}_{B N D}(G)$.

Theorem 4.3. For a connected BSVN graph $G, \operatorname{Per}_{B N D}(G)=G$ if and only if the BSVN detour eccentricity of each node of $G$ is $n-1, n=$ number of nodes in $G$.

Proof. Let $\operatorname{Per}_{B N D}(G)=G$. Then $\operatorname{Ecc}_{B N D}(p)=\operatorname{diam}_{B N D}(G), \forall p \in G$. So every node of $\mathrm{G}$ is a BSVN detour periphery node of G. Therefore G is a self-centered BSVN graph and $\operatorname{rad}_{B N D}(G)=\operatorname{diam}_{B N D}(G)=n-1$. So the BSVN detour eccentricity of each node of $\mathrm{G}$ is $n-1$.

Conversely, let the BSVN detour eccentricity of each node of $\mathrm{G}$ is $n-1$. So, $\operatorname{rad}_{B N D}(G)=\operatorname{diam}_{B N D}(G)=n-1$. All nodes of G are BSVN detour peripheral nodes and hence $\operatorname{Per}_{B N D}(G)=G$.

Theorem 4.4. For a connected BSVN graph $G, E c c_{B N D}(G)=G$ if and only if the BSVN detour eccentricity of each node of $G$ is $n-1, n=$ number of nodes in $G$.

Proof. Let, $\operatorname{Ecc}_{B N D}(G)=G$, So all nodes of G are BSVN detour eccentric node. Therefore, G is a self-centered BSVN graph and $\operatorname{diam}_{B N D}(G)=n-1$. Hence, the BSVN detour eccentricity of each node of $\mathrm{G}$ is $n-1$.

Conversely, let the BSVN detour eccentricity of each node of $\mathrm{G}$ is $n-1$. So $\operatorname{rad}_{B N D}(G)=\operatorname{diam}_{B N D}(G)=n-1$. So all nodes of G are BSVN detour peripheral nodes as well as BSVN detour eccentric nodes. Hence, $\operatorname{Ecc}_{B N D}(G)=G$.

Theorem 4.5. In a connected BSVN graph $G$, a node $b$ is a BSVN detour eccentric node if and only if $b$ is a BSVN detour peripheral node.

Proof. Let $\mathrm{b}$ be a BSVN detour eccentric node of G and let $b \in a_{B N D}^{*}$. Let $x$ and $y$ be two BSVN detour peripheral nodes, then $B N D(x, y)=\operatorname{diam}_{B N D}(G)=k(s a y)$. Let $P_{1}$ and $P_{2}$ be any $x-y$ and $a-b$ BSVN detour in G respectively. There arise two cases.

Case 1: When $b$ is not an internal node in G i.e, there is only one node, say $c$ which is adjacent to $b$. Soc $\in P_{2}$. Since G is connected, $c$ is connected to $a$ node of $P_{1}$, say $c^{\prime}$. So either $c^{\prime} \in P_{2}$ or $c^{\prime} \in\left(P_{1} \cap P_{2}\right)$. Thus, in any case the path from a to $\mathrm{x}$ or a to $\mathrm{y}$ through $\mathrm{c}$ and $c^{\prime}$ is longer than $P_{2}$. But it is impossible since $\mathrm{b}$ is a BSVN detour eccentric node of $a$. Hence $\operatorname{Ecc}_{B N D}(a)=\operatorname{diam}_{B N D}(G)$ i.e, $b$ is a BSVN detour peripheral node of $\mathrm{G}$.

Case 2: When $b$ is an internal node in $\mathrm{G}$, then there exists a connection between $b$ to $x$ and $b$ to $y$, because of the connectedness of G. Then $a-b$ BSVN detour can be extended to $x$ or $y$. This is impossible because $b$ is a BSVN detour eccentric node of $a$. Hence $\operatorname{Ecc}_{B N D}(a)=\operatorname{diam}_{B N D}(G)$ i.e, $b$ is a BSVN detour peripheral node of G.

Conversely, we assume that $b$ be a BSVN detour peripheral node G. So there exists a BSVN detour peripheral node say $a$ (distinct from $b)$. Therefore $b$ is a BSVN detour eccentric node of $a$.

\section{Conclusions}

In this study, we extended the part of BSVN sets to BSVN graphs. By Theorem 4.1, if $\operatorname{rad}_{B N D}(G)$ is equal to $\operatorname{diam}_{B N D}(G)$ and also BSVN graph is a BSVN detour is self-centered BSVN graph. Theorem 4.2 indicates rad $_{B N D}$ of G and diam $_{B N D}$ of G is equal to one less than the number of nodes of G. From theorem 4.3 and theorem 4.4 BSVN detour eccentricity is $n-1$, where $n$ is a number of nodes. Finally, Theorem 4.5 proves that any node is BSVN detour eccentric node based on BSVN detour peripheral node.

\section{References}

1) Broumi S, Smarandache F, Talea M, Bakali A. An Introduction to BSVN Graph Theory. Applied Mechanics and Materials. 2016;841:184-191. Available from: https://doi.org/10.4028/www.scientific.net/AMM.841.184.

2) Zadeh LA. Fuzzy sets. Information and Control. 1965;8(3):338-353. Available from: https://dx.doi.org/10.1016/s0019-9958(65)90241-x.

3) Atanassov KT. Intuitionistic fuzzy sets. Fuzzy Sets and Systems. 1986;20:87-96. Available from: https://dx.doi.org/10.1016/s0165-0114(86)80034-3.

4) Atanassov K, Gargov G. Interval valued intuitionistic fuzzy sets. Fuzzy Sets and Systems. 1989;31:343-349. Available from: https://dx.doi.org/10.1016/ 0165-0114(89)90205-4. 
5) Atanassov K. Intuitionistic fuzzy sets: Theory and Applications. New York. Physica. 1999. Available from: https://doi.org/10.1007/978-3-7908-1870-3_1.

6) Broumi S, Talea M, Bakali A, Smarandache F. On Bipolar Single Valued Neutrosphic Graphs. Journal of New Theory. 2016;11:84-102. Available from: https://doi.org/10.13140/RG.2.1.3354.0886.

7) Rao TSN, Kumar CS, Rao YS, Rao VV. Detour Interior and Boundary vertices of BSV Neutrosophic Graphs. International Journal of Advanced Science and Technology. 2020;29(8):2382-2394. Available from: http://sersc.org/journals/index.php/IJAST/article/view/23407.

8) Rao VV, Rao YS. Neutrosophic Pre-open Sets and Pre-closed Sets in Neutrosophic Topology. International Journal of ChemTech Research. 2017;10(10):449458.

9) Reddy GU, Rao TSN, Rao VV, Rao YS. Minimal Spanning tree Algorithms w. r. t. Bipolar Neutrosophic Graphs. London Journal of Research in Science Natural and Formal. 2020;20(8):13-24. Available from: https://journalspress.com/LJRS_Volume20/1264_Minimal-Spanning-Tree-Algorithms-w-r-tBipolar-Neutrosophic-Graphs.pdf.

10) Kumar CS, Rao TSN, Rao YS, Rao VV. Interior and Boundary vertices of BSV Neutrosophic Graphs. Journal of Advanced Research in Dynamical Control Systems. 2020;12(6):1510-1515. Available from: https://doi.org/10.5373/JARDCS/V12I2/S20201348.

11) Rao YS, Kumar CS, Rao TSN, Rao VV, Rao. Single Valued Neutrosophic detour distance. Journal of Critical Reviews. 2020;7(8):810-812. Available from: https://doi.org/10.31838/jcr.07.08.173. 